\title{
Computer Graphics
}

National Cancer Institute

\section{Source}

National Cancer Institute. Computer Graphics. NCI Thesaurus. Code C16460.

The field of visual computing, where one utilizes computers both to generate visual images synthetically and to integrate or alter visual and spatial information sampled from the real world. 